“C 2016 IEEE. Personal use of this material is permitted. Permission from IEEE must be obtained for all other uses, in any current or future media, including reprinting/republishing this material for advertising or promotional purposes, creating new collective works, for resale or redistribution to servers or lists, or reuse of any copyrighted component of this work in other works." 


\title{
An Extremely Wideband Tapered Balun for Application in Tightly Coupled Arrays
}

\author{
Alpha O. Bah ${ }^{1}$, Pei-Yuan Qin ${ }^{1}$, and Y. Jay Guo ${ }^{1}$
}

\begin{abstract}
This paper presents the design of a single layer, compact, tapered balun with a $>20: 1$ bandwidth and less than $\lambda / 17$ in length at the lowest frequency of operation. The balun operates from $0.7 \mathrm{GHz}$ to over $15 \mathrm{GHz}$. It can provide both impedance transformation as well as a balanced feed for tightly coupled arrays. Its performance is compared with that of a full-length balun operating over the same frequency band. There is a high degree of agreement between the two baluns.
\end{abstract}

\section{INTRODUCTION}

In recent years, the demand for extremely wideband (EWB), conformal, and thin arrays capable of more than 10:1 bandwidths has escalated. This demand is driven by bandwidth hungry systems such as software defined radios, radio telescopes, advanced radar systems, and electronic warfare systems [1]. As the platforms on which antennas operate continue to shrink, wideband multifunctional apertures employing multiple beams, polarizations, and frequency bands are needed. These apertures can consolidate various communications and sensing systems onto a single wideband device ensuring substantial reduction in size, weight, cost and power consumption [2].

To overcome some of the problems in existing wideband array designs and come up with systems that are compact, EWB, conformal and grating lobes free, new approaches to EWB array designs have been developed over the past few decades culminating in what is referred to as "Tightly Coupled Arrays" (TCA) [3, 4]. The advent of TCAs marked a revolutionary breakthrough in wideband array design in which capacitive mutual coupling is deliberately introduced between the array elements to cancel the effects of the ground plane inductance instead of modifying or suppressing ground plane reflections. This controlled mutual coupling combined with the non-resonant characteristic of the array, allows field propagation to neighbouring elements and eliminates impedance variations to give rise to large bandwidths in low profile implementations [5].

Although EWB TCAs have many desirable characteristics, several key challenges needs to be addressed for successful implementation. To practically realize very large bandwidths, an equally wideband feed network is required that can perform unbalanced to balanced as well as impedance transformations [6]. The feed network has to be of minimal size and cost and be able to handle high power levels.
Various feeding arrangements have been proposed in the literature for TCAs to produce a compact, low profile, low cost, and low insertion loss feed. These include differential feeds [7], printed baluns [8], tightly coupled dipole arrays with integrated baluns (TCDA-IB) [9], and Planar Ultrawideband Modular Array (PUMA) feeds [10]. The differential feeding method in conjunction with a resistive frequency selective surface (FSS) achieved the highest bandwidth (21:1) but employs lossy (2-7 dB) and bulky external $180^{\circ}$ hybrids. Printed Marchand baluns are compact and low profile but despite this, they yield reduced bandwidth (1.6:1) compared to other feeding methods and lower efficiency. The TCDA-IB helped overcome some of the limitations of the above-mentioned feeding methods. They use an integrated Marchand balun to obtain much better performance without the use of bulky external wideband baluns. However, they use a complicated structure with multiple layers and several vias to connect between layers. They also employ resistive FSS to achieve wider bandwidths but at the expense of lower gain and efficiency. The above mentioned feeds are not very scalable to the more promising area of programmable mm-wave front-ends because of the very tight tolerances required and the much smaller bandwidths $(2: 1)$ they offer at these higher frequencies. The PUMA feeding techniques have been developed that avoids baluns altogether and uses shorting posts to mitigate common mode resonances resulting in bandwidths of $6: 1(7.5-45$ $\mathrm{GHz}$ ) at mm-wave frequencies. However, these feeds require complex matching networks and could give rise to very high cross-polarization levels $(<8.5 \mathrm{~dB})$.

In this contribution, a single layer, low-profile tapered balun with a much reduced length and an extremely wide bandwidth $(>20: 1)$ is presented. It scales seamlessly from $\mathrm{MHz}$ to mm-wave frequencies and can transform between any two arbitrary impedances. Two designs are presented as a proof of concept for application in TCAs. The first design is a full size balun and the other is of a much reduced length that is about a fifth the size of the full size balun. The reduced balun achieves a very high level of current balance and a $180^{\circ} \pm 5^{\circ}$ differential phase shift at its output ports. The balun performance is very stable across the entire wideband with $<-10 \mathrm{~dB}$ return loss and $>-0.5 \mathrm{~dB}$ insertion loss.

\footnotetext{
${ }^{1}$ Faculty of Engineering and Information Technology, University of Technology Sydney, New South Wales, 2007, Australia; e-mail: alpha.bah@student.uts.edu.au.
} 


\section{BALUN DESIGN}

A full size balun called Balun A, and a shortened version called balun $\mathrm{B}$, were designed to operate from $0.7 \mathrm{GHz}$ to $>15 \mathrm{GHz}$. The structure of the designed baluns is shown in Figure 1 and their dimensions are given in Table 1 . The baluns can perform impedance transformation from the unbalanced $50 \Omega$ port at the input to the balanced 100 $\Omega$ port at the ouput. A $100 \Omega$ output port impedance was chosen to align with the input impedance of a TCA with two baluns per unit cell.

\subsection{Balun A}

Both the upper and lower conductors of the baluns are gradually tapered using a Klopfenstein taper [11] profile from the unbalanced input port to a balanced parallel strip of equal width at the output port. The characteristic impedance variation with distance along the taper is given as:

$$
\begin{aligned}
& \quad \ln Z(z)=\frac{1}{2} \ln \left(Z_{0} Z_{L}\right)+\frac{\Gamma_{0}}{\cosh A} A^{2} \phi\left(\frac{2 z}{L}-1, A\right) \\
& \text { for } 0 \leq z \leq L
\end{aligned}
$$

Where:

$$
\begin{gathered}
\phi(x, A)=-\phi(-x, A)=\int_{0}^{x} \frac{I_{1}\left(A \sqrt{1-y^{2}}\right.}{A \sqrt{1-y^{2}}} d y \\
\text { for }|x| \leq 1
\end{gathered}
$$

and $I_{1}(x)$ is the modified Bessel function. The resulting reflection coefficient of the taper is given as:

$$
\begin{aligned}
& \Gamma(\theta)=\Gamma_{0} e^{-j \beta L} \frac{\cos \sqrt{(\beta L)^{2}-A^{2}}}{\cosh A} \text { for } \beta L \geq A \\
& \Gamma(\theta)=\Gamma_{0} e^{-j \beta L} \frac{\cos \sqrt{A^{2}-(\beta L)^{2}}}{\cosh A} \text { for } \beta L<A
\end{aligned}
$$

In equations (1) $-(3), \Gamma_{0}$ is the reflection coefficient at zero frequency and it is given as:

$$
\Gamma_{0}=\frac{Z_{L}-Z_{0}}{Z_{L}+Z_{0}} \simeq \frac{1}{2} \ln \left(\frac{Z_{L}}{Z_{0}}\right)
$$

From (2), the maximum ripple in the passband $(\beta L \geq$ $A)$ is given as:

$$
\Gamma_{m}=\frac{\Gamma_{0}}{\cosh A}
$$

The minimum length of taper for a given $\Gamma_{m}$ in the passband can be calculated from equation (4). This length is approximately equal to half a wavelength at the lowest frequency of operation. Equations (1) - (4) were evaluated using MATLAB to obtain the impedance and reflection coefficient variation with distance along the taper. The closed form synthesis equations given in [12] were then used to calculate the physical dimensions of the taper width for all the impedance values, given the dielectric constant and substrate height. The resulting dimensions were imported into the commercial electromagnetic software, HFSS to model and optimize the design. A rogers RO4003 substrate with an $\varepsilon_{r}$ of 3.55 was used for balun $\mathrm{A}$.

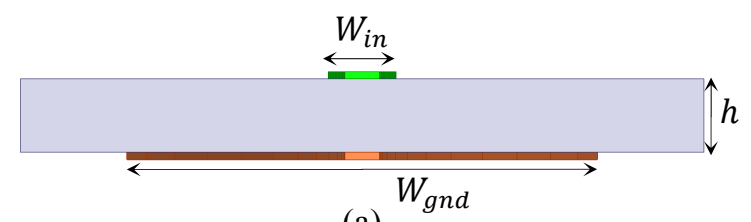

(a)

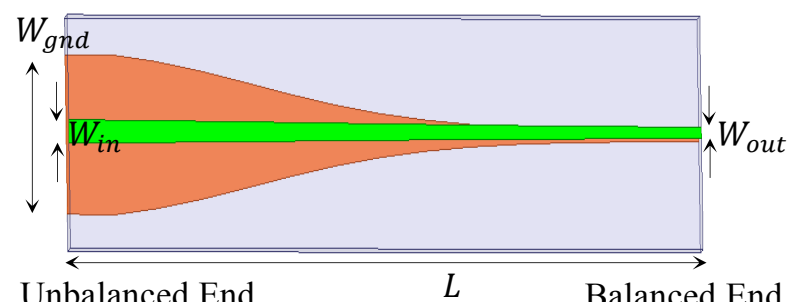

(b)

Figure 1: Tapered balun (a) side view (b) top view.

\begin{tabular}{|l|l|c|c|c|c|}
\hline & $W_{\text {gnd }}$ & $W_{\text {in }}$ & $W_{\text {out }}$ & $L$ & $h$ \\
\hline Balun A & 16.66 & 3.41 & 1.698 & 133.59 & 1.524 \\
\hline Balun B & 3.33 & 0.47 & 0.237 & 25.25 & 0.508 \\
\hline
\end{tabular}

Table 1: Dimensions of baluns A and B (in mm).

\subsection{Balun B}

TCAs require balun feeds that are approximately $0.45 \lambda$ at the highest frequency of operation. For an array operating up to $5 \mathrm{GHz}$, the full size balun is about five times longer than required. In order to reduce the length of the taper, the number and size of the transformer sections used to approximate the continuous taper were reduced and optimized for a smoother transition and for minimal return loss in the passband. The resulting balun has a performance very much identical to that of the full size balun. A rogers RT duroid 6010 substrate with a higher $\varepsilon_{r}$ of 10.2 was used to further reduce the balun size. A thinner substrate was also employed to minimize the effect of surface waves in the array.

\section{RESULTS AND DISCUSSION}

The simulated results comparing the performance of the two baluns are presented in figures 2 and 3 . In figure 2, the $S_{21}$ of balun $A$ varies between $-0.38 \mathrm{~dB}$ and $-0.81 \mathrm{~dB}$ whereas that of balun $B$ varies between $-0.5 \mathrm{~dB}$ and $-0.24 \mathrm{~dB}$. Balun A has a slightly better insertion loss at the lower frequency band and balun $\mathrm{B}$ has a slightly better insertion loss at the higher frequency band. The $S_{11}$ of balun $A$ has a much sharper drop compared to the gradual drop of Balun B. However, the $S_{11}$ of balun $B$ is still maintained between $-11 \mathrm{~dB}$ and $<-30 \mathrm{~dB}$ across the band. We 
can see that the baluns' performance improves as the frequency increases and a bandwidth in excess of $20: 1$ is achieved. Despite the reduced length of balun $\mathrm{B}$, its performance is virtually identical to that of the full size balun. The common mode rejection ratio (CMRR) of balun B was also calculated in HFSS and is shown in figure 1 . The worst CMRR is $-25 \mathrm{~dB}$ extending to $-40 \mathrm{~dB}$ at the higher frequency of the band. This value of common mode rejection ensures that the disastrous common modes usually encountered in the array environment are avoided. The worst case CMRR reported here is about $7 \mathrm{~dB}$ better than that reported in [13].

The amplitude and phase balance at the output ports of the two baluns are shown in figure 3 . The amplitude balance of both baluns oscillates around the ideal value of one. The phase balance of balun $\mathrm{A}$ is $180^{\circ} \pm 5^{\circ}$ and that of Balun B is $180^{\circ} \pm 10^{\circ}$. The difference in phase between the two baluns mostly occur between $0 \mathrm{GHz}$ and $2.5 \mathrm{GHz}$ and are comparable for the rest of the band.

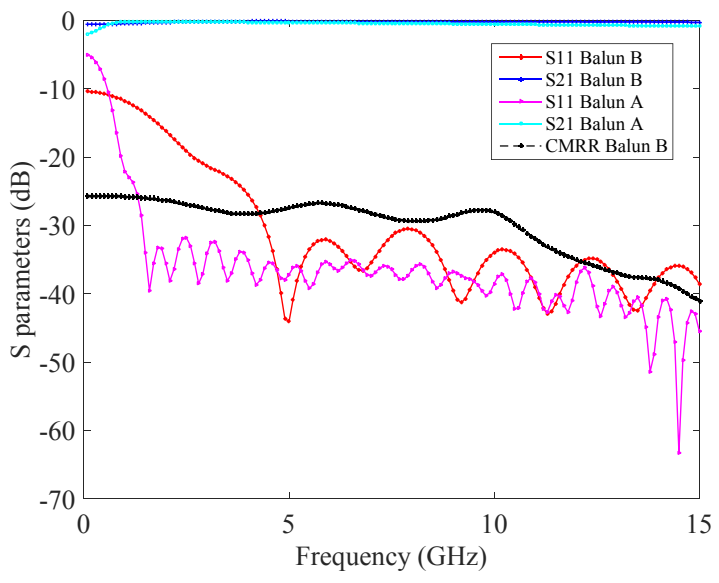

Figure 2: S-parameters of baluns A and B.
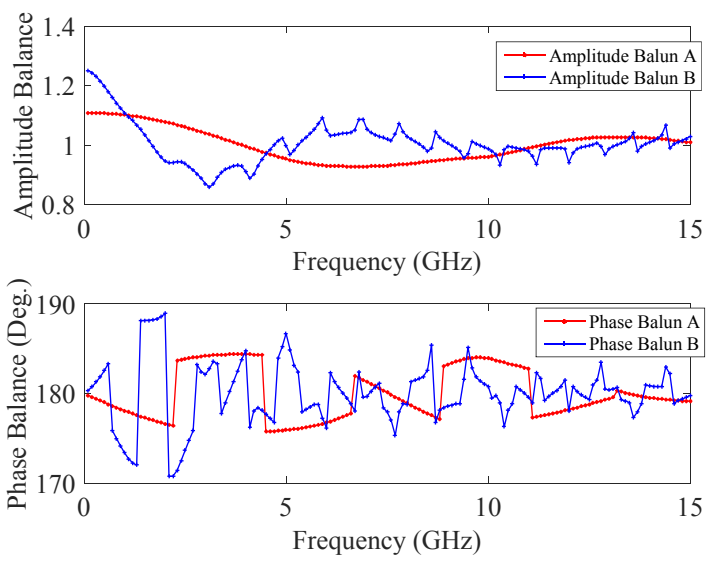

Figure 3: Amplitude and phase balance of baluns A and $\mathrm{B}$.

\section{CONCLUSIONS}

A single layer, low-profile tapered balun with a much reduced length and an extremely wide bandwidth ( $>$ 20:1) was designed as a feed for TCAs. A full size tapered balun was also designed whose length was approximately five times that of the reduced length balun. The performance of the reduced length balun was comparable to that of its full size counterpart in terms of current balance, phase balance, bandwidth, common mode rejection, return loss and insertion loss. The performance of the reduced length balun compares favourably to other baluns in the literature with the added benefits of simplicity and compactness.

\section{Acknowledgments}

The Authors would like to acknowledge the support of both the Coorporate Research Centre for Space Environment Management (SERC Limited) through the Australian Government's Coorporative Research Centre Programme and the Australian Research Council under ARC DP160102219 grant.

\section{References}

[1] G. C. Tavik, C. L. Hilterbrick, J. B. Evins, J. J. Alter, J. G. Crnkovich, and J.W. De Graaf et al, "The advanced multifunction RF concept," IEEE Trans. Microwave Theory and Techniques, vol. 53, no. 3, pp. 1009-1020, Mar. 2005.

[2] J. J. Lee, "Ultra wideband arrays," in Antenna Engineering Handbook, 4th ed., J. L. Volakis, Ed. McGraw Hill Professional, 2007.

[3] B. A. Munk, Finite Antenna Arrays and FSS, ch. 6, pp. 181-213, John Wiley \& Sons, Hoboken, NJ, 2003.

[4] M. Jones and J. Rawnick, "A new approach to broadband array design using tightly coupled elements," in Proc. IEEE MILCOM, pp. 1-7, Oct. 29-31, 2007.

[5] W. F. Croswell, T. Durham, M. Jones, D. Schaubert, P. Friederich, and J. G. Maloney, "Wideband arrays," in Modern Antenna Handbook, C.A. Balanis, Ed. New York: WileyInterscience, pp. 581-627, 2008.

[6] I. Tzanidis, K. Sertel, and J. L. Volakis, "UWB Low-Profile Tightly Coupled Dipole Array With Integrated Balun and Edge Terminations," in IEEE Transactions on Antennas and Propagation, vol. 61, no. 6, pp. 3017-3025, Jun 2013.

[7] W. F. Moulder, K. Sertel, and J. L. Volakis, "Superstrate-Enhanced Ultrawideband Tightly Coupled Array With Resistive FSS," in IEEE Transactions on Antennas and Propagation, vol. 60, no. 9, pp. 4166-4172, Sep. 2012. 
[8] J. A. Kasemodel and J. L. Volakis, "A Planar Dual Linear-Polarized Antenna With Integrated Balun," in Antennas and Wireless Propagation Letters, vol. 9, pp. 787-790, 2010.

[9] J. P. Doane, K. Sertel, and J. L. Volakis, "A Wideband, Wide Scanning Tightly Coupled Dipole Array With Integrated Balun (TCDA-IB)," in IEEE Transactions on Antennas and Propagation, vol. 61, no. 9, pp. 4538-4548, Sep. 2013.

[10] S. S. Holland and M. N. Vouvakis, "The Planar Ultrawideband Modular Antenna (PUMA) Array," in IEEE Transactions on Antennas and Propagation, vol. 60, no. 1, pp. 130-140, Jan. 2012.

[11] R. W. Klopfenstein, "A Transmission Line Taper of Improved Design," Proceedings of the IRE, vol. 44, pp. 31-15. Jan. 1956.

[12] D. M. Pozar, Microwave Engineering, ch. 3, pp. 148-149, John Wiley \& Sons, Hoboken, NJ, 2012.

[13] D. Cavallo, A. Neto and G. Gerini, "PCB Slot Based Transformers to Avoid Common-Mode Resonances in Connected Arrays of Dipoles," in IEEE Transactions on Antennas and Propagation, vol. 58, no. 8, pp. 2767-2771, Aug. 2010. 